\title{
IMPROVING THE SPATIAL RESOLUTION OF IMAGING INSTRUMENTS
}

USING SOFTWARE

Manohar Mareboyana ${ }^{1,2}$, Jacqueline Le Moigne ${ }^{3}$, Philip Dabney ${ }^{4}$

Bowie State University, Bowie MD 20715

ASRC at NASA Goddard, Greenbelt, MD 20771

Software Engineering Division, NASA Goddard, Greenbelt, MD 20771

Biospheric Sciences Lab, NASA Goddard, Greenbelt, MD 20771

\section{ABSTRACT}

In order to overcome spatial resolution limitations associated with physical sensor limitations when using SmallSats and CubeSats, we utilize an image processing technology referred to as Super-Resolution (SR). In general, software approaches are increasingly considered in connection with smaller satellites for which size, mass and power constraints limit the sensor capabilities. Being able to perform hardware vs. software trades might enable more capabilities for a lowel cost. This paper describes recent experiments conducted to optimize the spatial enhancement of acquired observations using multiple sub-pixel shifted low resolution images

\section{SCIENCE REQUIREMENTS}

With many future missions planning to use CubeSats and SmallSats, software approaches are increasingly considered to alleviate the size constraints of these platforms that limit the sensor capabilities. For example, the most common CubeSat sizes are $3 \mathrm{U}$ and $6 \mathrm{U}$, effectively limiting apertures and pupils to approximately $9 \mathrm{~cm} \times 9 \mathrm{~cm}$ and possibly an ellipsoid of $\sim 9 \mathrm{~cm} \times 18 \mathrm{~cm}$. This produces a hard cutoff of spatial frequencies above 1 line/ 2.5 meters with a steep rolloff leading up to that point.

Furthermore, most low-power fine-pitch focal planes with high frame rates have low fill-factors when micro-lens arrays are eliminated to maximize the detector numerical aperture (NA) for fast optical systems and utilize the small instantaneous field of views (IFOVs) the small detector areas create. This low fill-factor produces an instantaneously under-sampled and aliased image. Super-Resolution (SR) seeks to recover the higher resolution information that produces the alias and place the energy back in the appropriate location. It does this by intentionally moving the under-sampled alias image in sub-pixel pitch increments to capture all of the spatial energy delivered to the focal plane from multiple exposures of the same scene that differ in subpixel shifts (Fig 1).

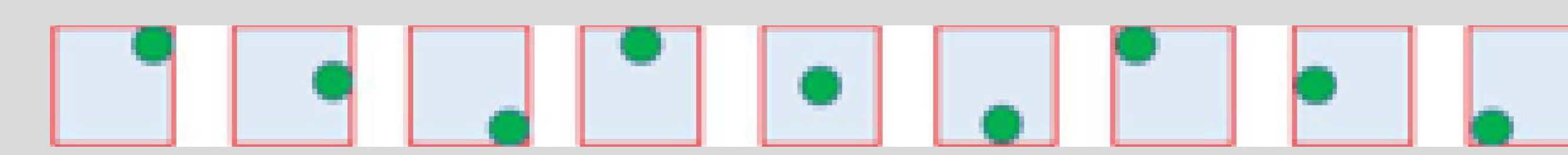

Figure 1 - Example of super-resolution algorithm using 9 input images that differ in subpixel shifts. The middle pixel being reference

\section{SR - ALGORITHMIC APPROACH:}

Frequency Domain Approach [2] with the following characteristics:

Computationally efficient (using Discrete or Continuous

Fourier Transform and aliasing properties to combine Low-

Resolution, LR, images in the SR algorithm)

Regularization complicated as image degradation models

become complex

Spatial Domain Approaches with the following specific methods:

Non-Iterative approaches including interpolation and restoration:

Radial Basis Function (RBF)

Inverse Distance Weighted (IDW)

Nearest Neighbor (NN)

Iterative Back Projection (IBP) [3]

Statistical Approaches such as:

Maximum A posterior (MAP)

Maximum Likelihood (MLE)

\section{SR - SIMULATION AND VALIDA}

Ideally test images will be created from very high-resolution (HR) images such as Worldview-1 or Worldview-2, although any image at a reasonable resolution could be used in that framework. In a first step, the original HR image is being transformed by a number of sub-pixel shifts to create the HR shifted images $\left\{\mathrm{HR}_{\mathrm{S} 1}, \mathrm{HR}_{\mathrm{S} 2}, \ldots, \mathrm{HR}_{\mathrm{Sn}}\right\}$. Then the Point Spread Function (PSF) of the instrument being targeted is applied to each of these $\mathrm{HR}_{\mathrm{Sk}}$ images. The next step is then to downsample each of the resulting images by the amount of resolution enhancement the super-resolution algorithm is being validated for, thus creating the low-resolution (LR) images that the $\mathrm{SR}$ algorithm will work from or $\left\{\mathrm{LR}_{\mathrm{S} 1}, \mathrm{LR}_{\mathrm{S} 2}, \ldots, \mathrm{LR}_{\mathrm{Sn}}\right\}$. used for the reconstruction.

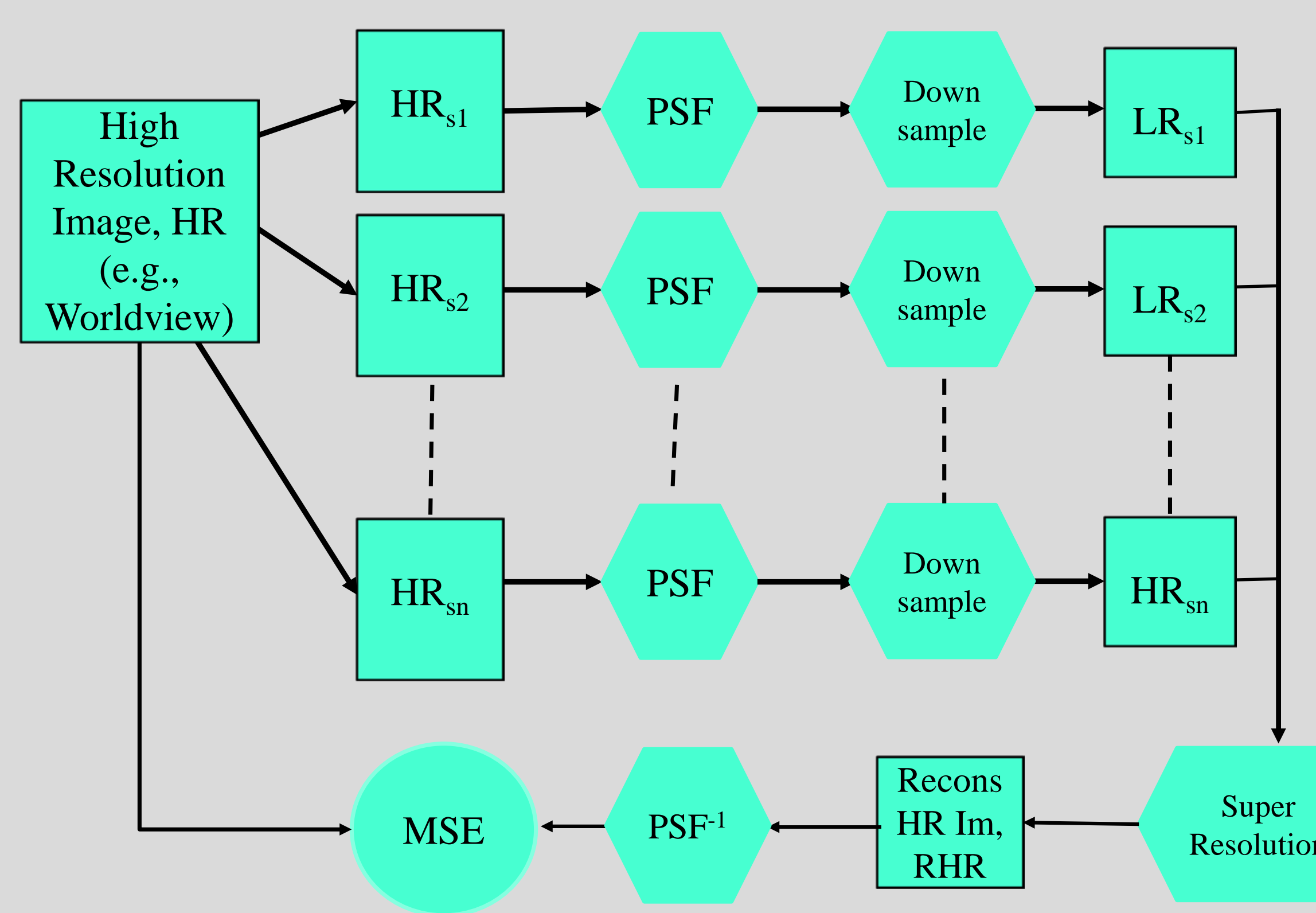

Figure 2 - Super Resolution Algorithm Validation Framework RADIAL BASIS FUNCTION (RBF) AND EDGE-DIRECI RBF (EDRBF)

Based on the previous results, our work then focused on the RBF technique and an extension of this method exploiting the directional information of edges to further improve the accuracy of RBF, the Edge-Directed Radial Basis Function (EDRBF) interpolation. The accuracy of SR depends on various factors besides the algorithm (i) number of sub-pixel shifted LR images (ii) accuracy with which the LR shifts are estimated by registration algorithms (iii) and the targeted spatial resolution of SR. In our studies, the accuracy of RBF and EDRBF will be compared with other algorithms keeping these factors constant.

RBFs are real valued functions whose value depends on the distance from the origin

$\emptyset(x, x i)=\emptyset\left(\left\|x-x_{i}\right\|\right)$--------(2)

Interpolated pixels $\mathrm{Z}(\mathrm{x}, \mathrm{y})$ values are determined from shifted LR images, $\operatorname{LR}_{\mathrm{k}}(\mathrm{x}, \mathrm{y})$ as follows

$Z(x, y)=\sum_{(k, i)} L R_{k}(x i, y i) \emptyset\left(\left\|(x, y)-\left(x_{i}, y i\right)\right\|\right)$

RBF is a Gaussian function

$$
\varnothing(r)=e^{-\gamma r}
$$

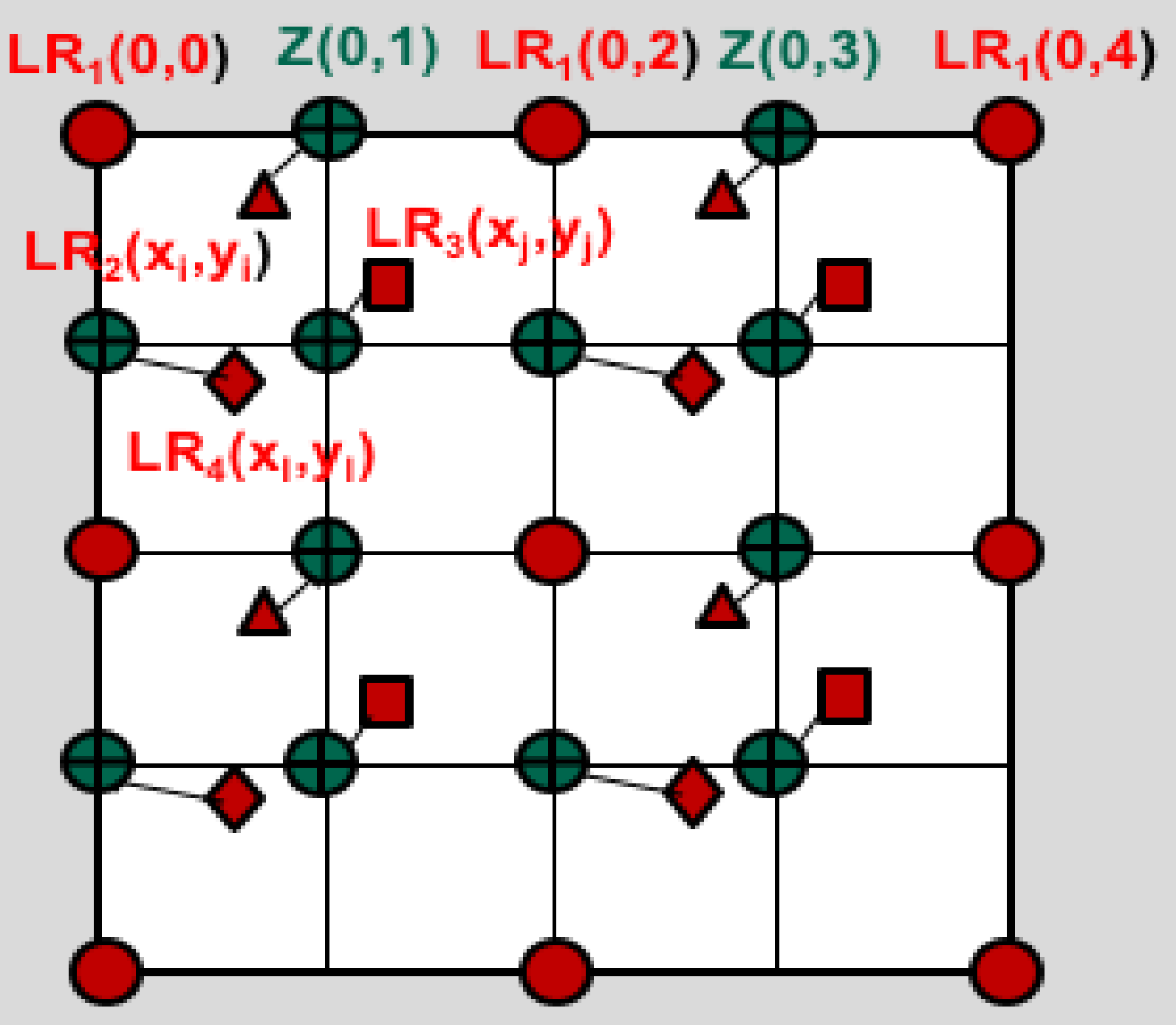

$\mathrm{LR}_{1}$ Pixels (Reference)

$\Delta \square \mathrm{LR}_{\mathrm{k}}$ Pixels with SubPixel Shifts

Z Interpolated Pixels

Figure 3 - Radial Basis Functions are used to compute interpolated pixels (in green) from the subpixel values given by the multiple LR images (in red)

\section{PERFORMANCE COMPAFIS}

\section{SR ALGORITHMS}

\begin{tabular}{|l|l|l|l|l|}
\hline \multirow{2}{*}{ Method } & \multicolumn{2}{l|l}{ Experiment 1 } & \multicolumn{2}{l|}{ Experiment 2} \\
\cline { 2 - 5 } & MSE & PSNR (dB) & MSE & PSNR(dB) \\
\hline $\begin{array}{l}\text { NN(Nearest Neighbor) } \\
\text { Interpolation }\end{array}$ & 3.16 & 38.81 & 5.43 & 40.78 \\
\hline $\begin{array}{l}\text { IDW(Inverse Distance } \\
\text { Weighted) }\end{array}$ & 3.18 & 38.78 & 5.47 & 40.75 \\
\hline $\begin{array}{l}\text { MLE (Maximum } \\
\text { Likelihood) }\end{array}$ & 3.79 & 38.02 & 4.7 & 41.40 \\
\hline $\begin{array}{l}\text { IBP (Iterative Back } \\
\text { Projection) }\end{array}$ & 4.14 & 37.63 & 6.12 & 40.26 \\
\hline $\begin{array}{l}\text { RBF } \\
\text { EDRBF }\end{array}$ & 1.28 & 42.73 & 1.53 & 46.28 \\
\hline
\end{tabular}

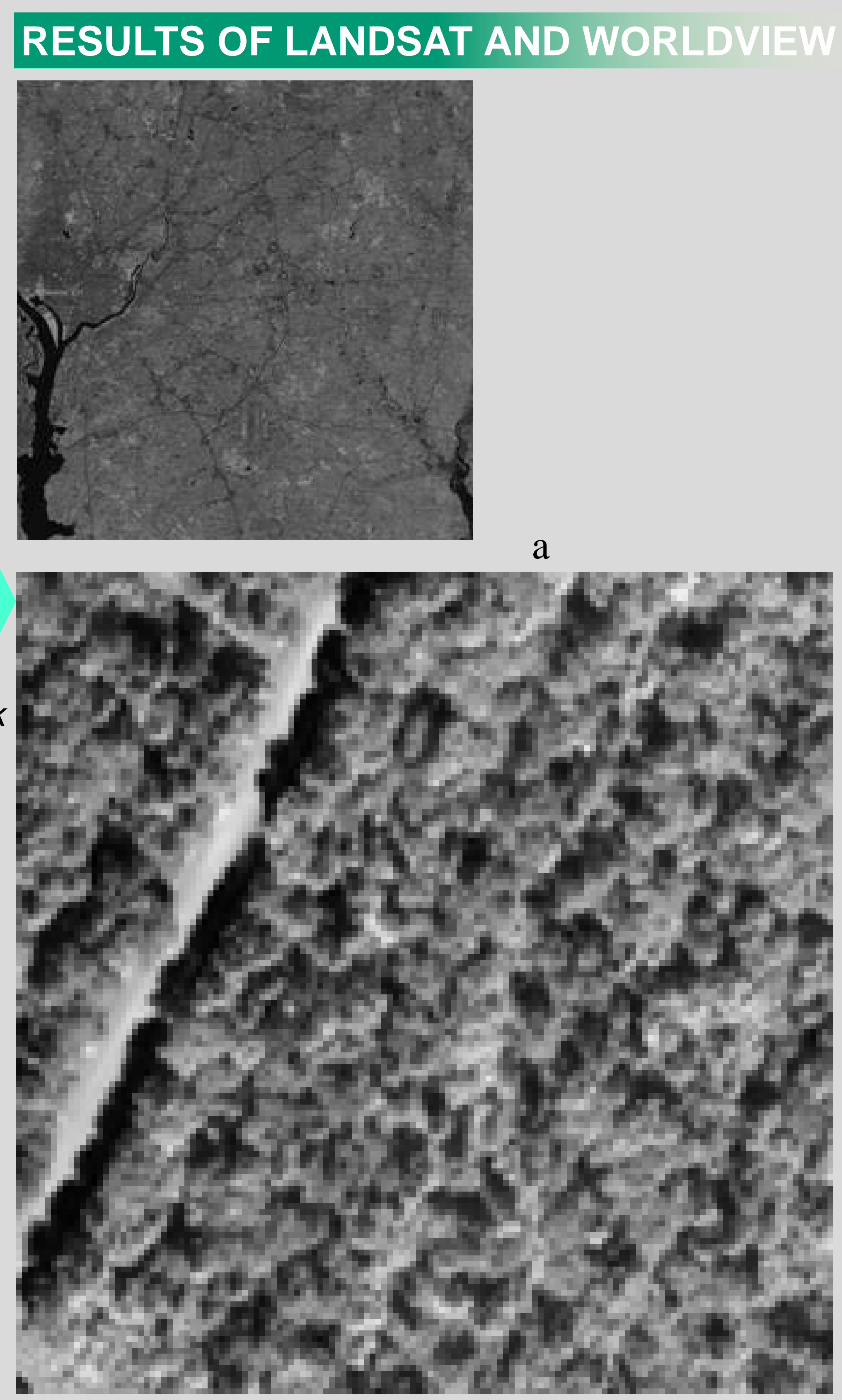

Figure 4 - Test images (a) Landsat (b) World view
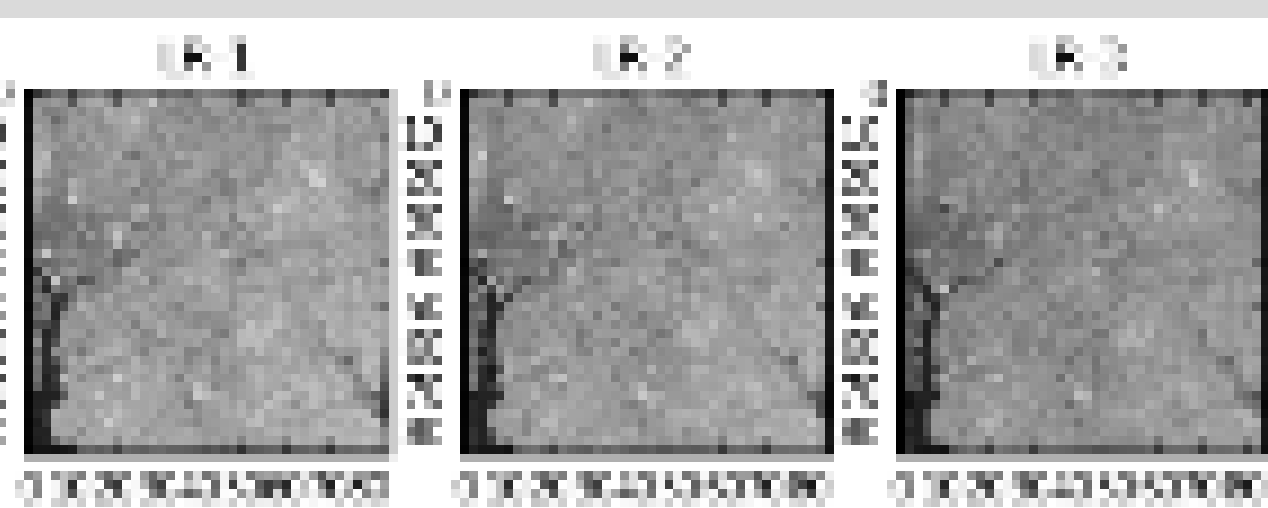

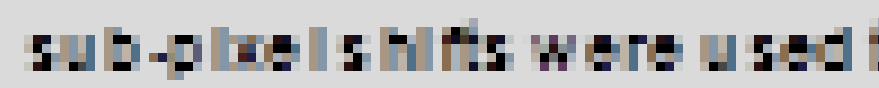
redes Laimages

$00-00=-10-00$

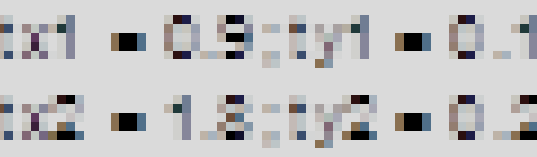
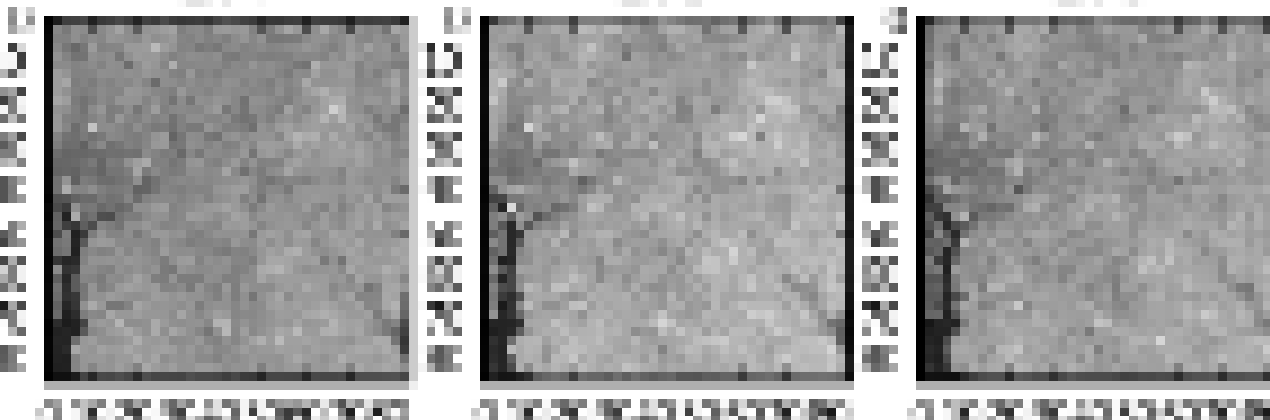

0 - o $0=-130$

$x+-09=4-10$
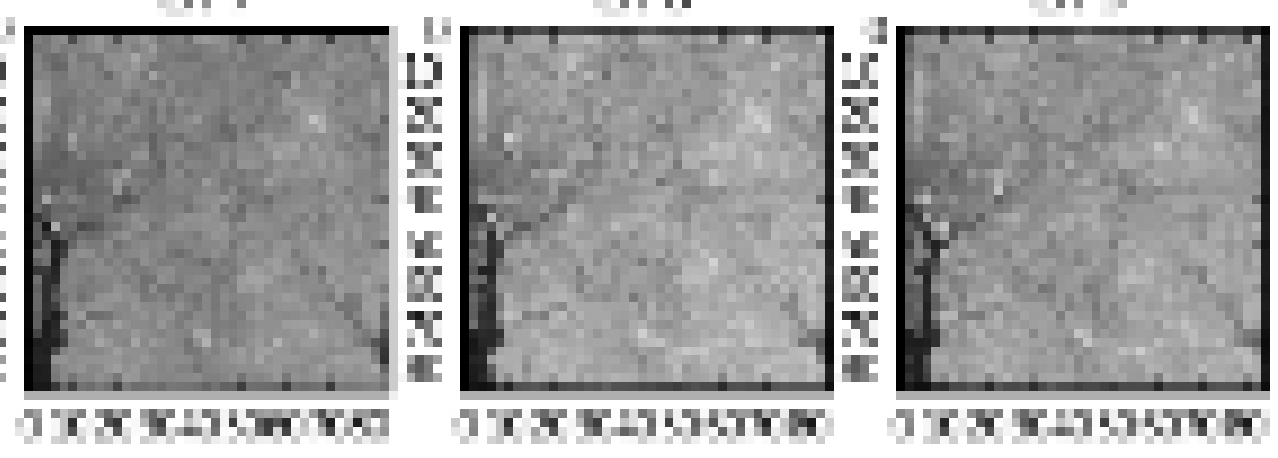

$0-03-16-2$

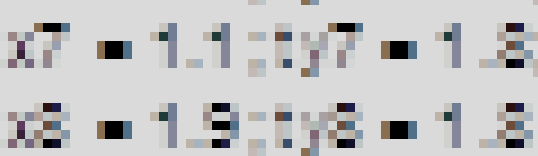

Figure 5 - Nine 90m LR images simulat

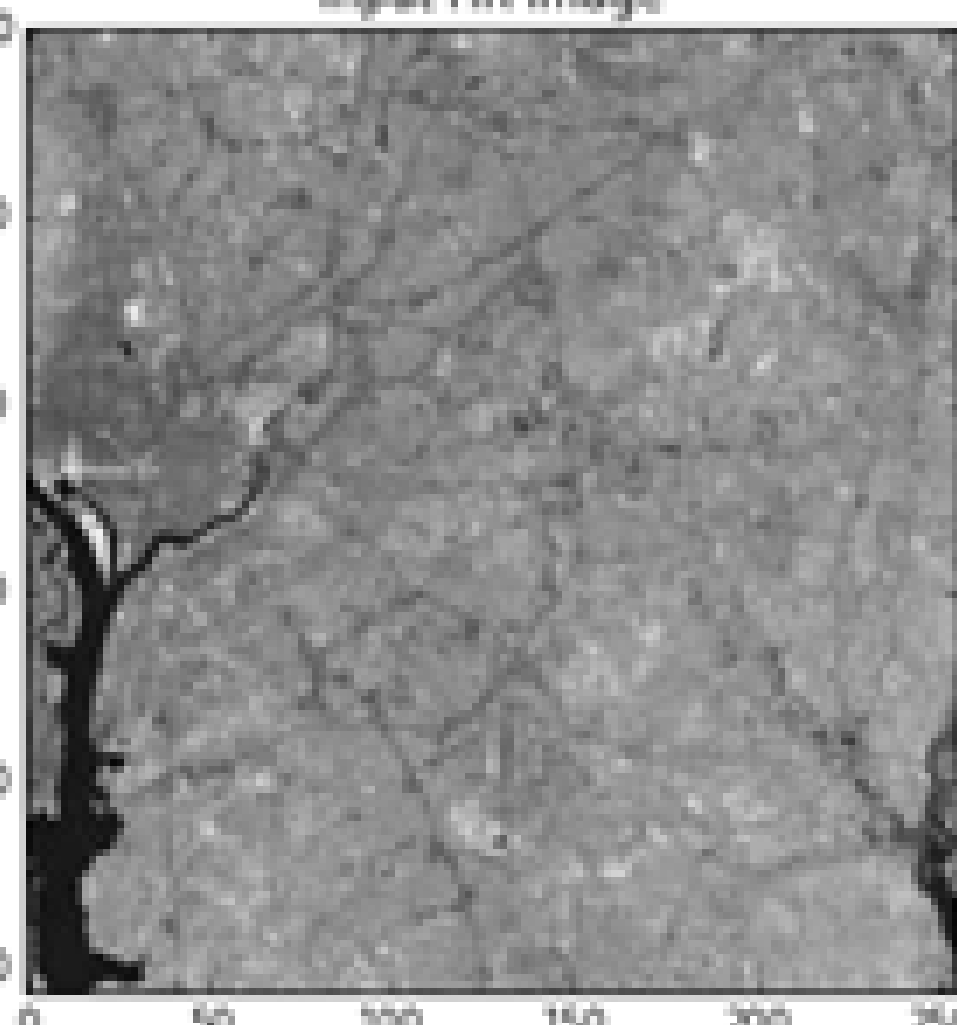

In put HR Landsat Image $\Sigma 6 \times \Sigma_{6}$

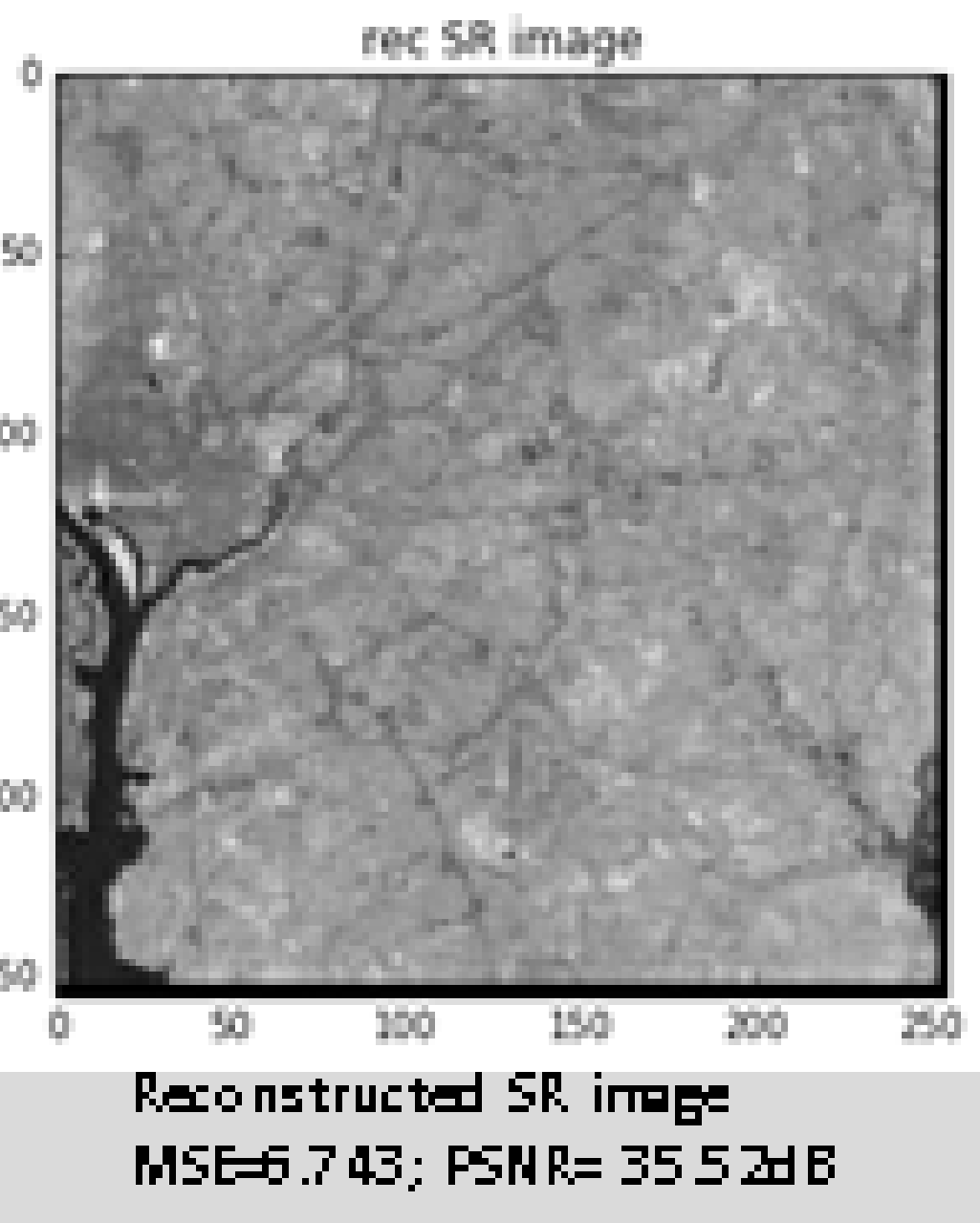

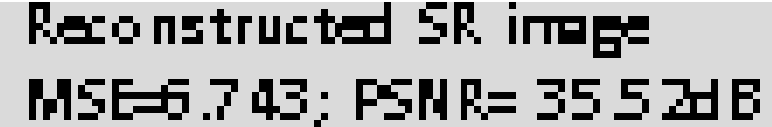

Figure 6 - Comparing original(left) reconstructed (right) Landsat images
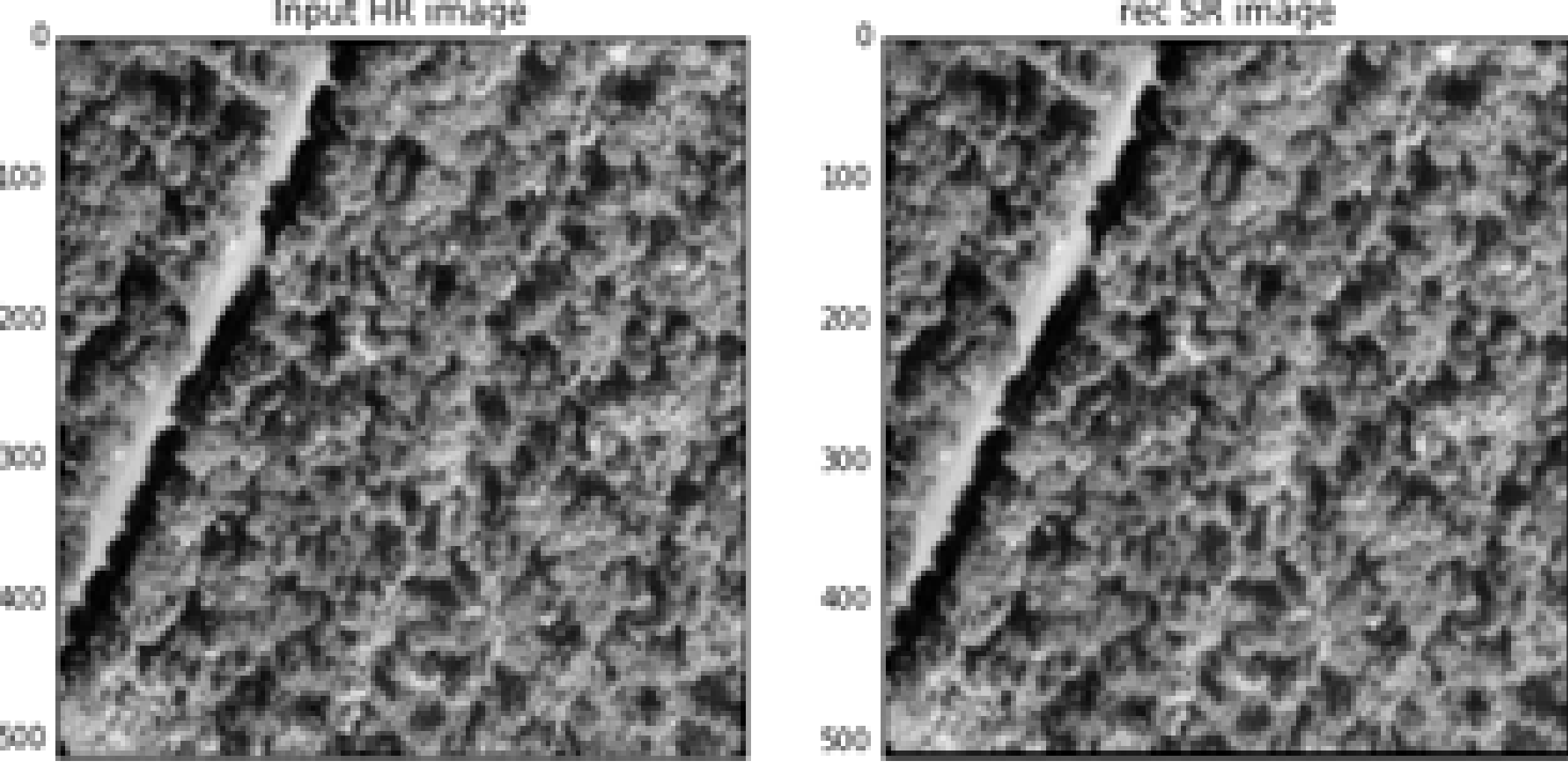

Figure 7 - Comparing original(left) reconstructed (right) Worldview images 